\title{
Do acolhimento ao encaminhamento: $O$ atendimento às tentativas de suicídio nos contextos hospitalares
}

Ana Paula Araújo de Freitas. Universidade Federal de Santa Catarina. Lucienne Martins Borges. Universidade Federal de Santa Catarina.

\section{Resumo}

Este estudo teve como objetivo investigar o acolhimento, o atendimento e os encaminhamentos feitos aos usuários atendidos por tentativa de suicídio, em duas urgências e duas emergências hospitalares de um município do Sul do Brasil. De caráter qualitativo, foram entrevistados 16 profissionais de saúde de nível superior, de diferentes categorias profissionais. Os resultados mostraram o predomínio do atendimento ao sexo feminino, as intoxicações como método mais frequente e o uso da tentativa de suicídio como forma de chamar a atenção. Ainda, evidenciou-se a existência de protocolos mínimos de atendimento, que, contudo, apresentam dificuldades nos encaminhamentos pós-alta para a rede de saúde. Ressalta-se, assim, a necessidade de capacitação dos profissionais para lidar com esta demanda bem como de melhor articulação do Sistema de Saúde e outras políticas para acolher os usuários após sua alta.

Palavras-chave: tentativa de suicídio e contexto hospitalar; saúde pública; dor psíquica.

\begin{abstract}
From reception to triage: Care of suicide attempts in hospital emergencies. This study aimed to investigate the reception, care and referrals given to users attended for suicide attempts in two urgency and two emergency services of a city in the south of Brazil. Qualitatively, sixteen health professionals of higher education from different professional categories were interviewed. The results show the predominance of the female sex, intoxication as the most frequent method and the use of the attempted suicide as a way to call attention. The existence of minimum care protocols is perceived. There is, however, a difficulty in post-discharge referrals to other health services. Thus, it highlights the need of capacitation for professionals to deal with this demand and a better articulation between the Health System and other policies to receive users after discharge as well.
\end{abstract}

Keywords: suicide attempted and hospital setting; public health; psychic pain.

\section{Resumen}

De la recepción a derivación: Intentos de suicidio en las urgencias hospitalarias. Este estudio tuvo como objetivo investigar la recepción, atención y derivación de usuários atendidos por intento de suicidio en dos servicios de emergencia y dos servicios de urgencias de un gran municipio del sur de Brasil. De caracter cualitativo, fueron encuestados dieciséis profesionales de salud de nivel superior, de categorias distintas. Los resultados muestran el predominio del sexo femenino, la intoxicación como método más frecuente y el intento de suicidio como una forma de llamar la atención. También pone de relieve la existencia de protocolos mínimos de atención, sin embargo, al post alta hay dificultades para las derivaciones a la red. Así, destaca la necesidad de capacitar a los profesionales para manejar esta demanda, así como articular mejor el sistema de salud y otras políticas para dar cabida a los usuarios al post alta. Palabras clave: intento de suicídio e contexto hospitalário; salud publica; dolor psíquico. 
As tentativas de suicídio, entendidas como o ato deliberado de tirar a própria vida, porém sem consumá-lo (Ministério da Saúde, 2009), são um importante problema para a Saúde Pública (Organização Mundial da Saúde [OMS], 2014; Vidal \& Gontijo, 2013). Dados da OMS apontam que no ano de 2012, aproximadamente 800 mil pessoas tiraram suas próprias vidas (OMS, 2014), contudo estima-se que para cada suicídio, um número 10 a 20 vezes maior de tentativas são praticadas. No Brasil, as taxas de suicídio são consideradas baixas quando comparadas à média mundial (4,1 mortes/100.000 hab no Brasil e 11,4 mortes/100.000 hab no mundo), porém o aumento absoluto de 33,5\% no número de mortes entre 1998 e 2008 tem chamado a atenção para a dimensão do problema (Waiselfisz, 2011).

Estudos apontam a predominância de mortes por suicídio entre homens e de tentativas entre mulheres, bem como a presença de métodos como enforcamento e armas de fogo na população masculina e intoxicação de medicamentos na feminina (Bando, Brunoni, Fernandes, Benseñor, \& Lotufo, 2012; Sá et al., 2010). A alta taxa de mortalidade entre homens está relacionada à letalidade do método utilizado, o que explica o elevado número de atendimentos ao público feminino nos ambientes hospitalares (Magalhães et al., 2014; Vidal, Gontijo, \& Lima, 2013). Ainda, a presença de transtornos mentais e histórico de tentativas de suicídio são apontados como fatores de risco importantes para novas tentativas (Botega, 2015; Stefanello \& Furlanetto, 2012).

Dado o aumento expressivo nas taxas de suicídio e tentativas, é crescente a demanda por atendimento a estas situações nos contextos hospitalares. Porém, o estudo de Botega et al., (2009) revelou que apenas uma em cada três pessoas que tentam suicídio é atendida em um serviço médico hospitalar. Implicam na procura por este recurso aspectos como gravidade da lesão autoprovocada e risco de morte, facilidade de acesso ao serviço de saúde e confiança do usuário no sistema, estigma das demais pessoas em relação ao seu comportamento e medo de ser criminalizado. De maneira geral, é um número reduzido frente às estimativas de tentativas que são praticadas (Botega et al., 2009; Nock et al., 2008).

A atitude dos profissionais de saúde é uma variável importante nesta procura. Estudos apontam atitudes negativas dos profissionais de saúde no atendimento às tentativas de suicídio (Silva \& Boemer, 2004; Vidal \&
Gontijo, 2013). Consumo de tempo desnecessário que poderia ser dedicado a pacientes mais graves, em especial quando há baixo risco de morte, raiva, irritação, descaso, negação ou a avaliação de que a demanda não é legítima para as emergências hospitalares, são aspectos apontados na literatura e que impactam negativamente no cuidado ofertado aos pacientes. (Freitas \& Martins-Borges, 2014; Srivastava \& Tiwari, 2012). Desta forma, entende-se que a relação do paciente com o profissional de saúde, da acolhida até a saída do serviço, é um importante instrumento para a continuidade ou não dos encaminhamentos realizados, bem como para a prevenção de novas tentativas de suicídio (Vidal \& Gontijo, 2013).

Nesse sentido Gutierrez (2014) assinala que um acolhimento de qualidade realizado no serviço de saúde pode determinar que o paciente aceite e dê continuidade ao seu tratamento. Para tal, este acolhimento deve oferecer atenção resolutiva às demandas e a articulação necessária, caso haja necessidade de continuidade do tratamento (Ministério da Saúde, 2013). Somado ao atendimento e aos encaminhamentos, estes três momentos constituem um fluxo de trabalho cuja tarefa é dar conta das diferentes necessidades de saúde/doença trazidas pelo paciente (Gutierrez, 2014). Todavia, ainda que o espaço das emergências configure-se como um local privilegiado para o cuidado e também para a avaliação do risco de novas tentativas, este nem sempre é aproveitado em todas as suas potencialidades e os pacientes são liberados sem a avaliação de um psiquiatra ou sem qualquer outro tipo de encaminhamento (Vidal \& Gontijo, 2013).

Observa-se, portanto, a necessidade de incremento na produção científica sobre a temática apresentada, uma vez que uma parcela importante dos estudos sobre a mesma tem se debruçado na discussão de aspectos epidemiológicos, de gênero e sobre a presença de fatores de risco, como transtornos mentais ou uso de drogas presentes nas tentativas de suicídio (Bando et al., 2012; Santos, Legay, \& Lovosi, 2013). Assim, entendendo a necessidade da prestação de uma assistência adequada às pessoas que tentaram suicídio, seja por meio da qualificação da prática profissional ou do aprimoramento de políticas públicas que levem a um cuidado mais humanizado, este artigo se propõe a investigar o acolhimento, o atendimento e os encaminhamentos realizados por profissionais de saúde a usuários atendidos após tentativa de suicídio em urgências e emergências hospitalares. 
Do acolhimento ao encaminhamento: $\mathrm{O}$ atendimento às tentativas de suicídio nos contextos hospitalares

\section{Método}

\section{Local}

Esta pesquisa foi realizada em duas urgências e duas emergências hospitalares da rede pública de um município do sul do país. As urgências hospitalares são compostas pelas UPAs (Unidades de Pronto Atendimento), que são estruturas de complexidade intermediária e funcionam 24h por dia, situadas entre as Unidades Básicas de Saúde e urgências hospitalares - os prontos-socorros (Ministério da Saúde, 2012). As emergências hospitalares, comumente chamadas de prontos-socorros, são os locais onde são atendidas as situações clínicas nas quais o paciente apresenta risco imediato e necessita de atendimento igualmente imediato, seja para estabilização das funções vitais ou para o alívio da dor (Simonetti, 2004).

\section{Participantes}

Realizou-se esta pesquisa com 16 profissionais com diferentes formações na área da saúde, entre eles médicos, psicólogos, enfermeiros e assistentes sociais. Oito trabalhavam em serviços de urgência (UPAs) e oito em serviços de emergência hospitalar (hospitais gerais). A escolha do número de participantes baseou-se no critério da saturação dos dados, quando se observa no decorrer das entrevistas que os elementos temáticos passam a se repetir e há poucos acréscimos aos já existentes, situação esta observada a partir da $13^{a}$ entrevista. Os critérios de inclusão considerados foram a experiência no atendimento a tentativas de suicídio, ou seja, o profissional já teve sob seus cuidados pelo menos um paciente que acessou a unidade por tentativa de suicídio; e a atuação nos serviços de urgência e emergência hospitalares, no momento da pesquisa.

\section{Instrumentos}

Neste estudo, descritivo e de caráter qualitativo, o instrumento utilizado para acessar as informações foi um roteiro de entrevistas composto por 11 questões, que tinha como objetivo nortear o discurso do participante. Tais questões investigavam a prática profissional dos participantes junto aos pacientes desde o acolhimento, as rotinas de atendimento e os encaminhamentos realizados após a alta. Utilizou-se também um questionário sócio-demográfico cujos itens contemplados foram o sexo e a idade do participante, tempo de exercício da profissão e tempo de trabalho em urgência ou emergência, número estimado de tentativas atendidas pelo profissional e a oferta de capacitação sobre a temática por parte das instituições nas quais trabalhavam.

\section{Procedimentos de Coleta de Dados}

A coleta de dados aconteceu entre os meses de maio e julho de 2013 e o convite aos participantes se deu por contato telefônico, a partir de uma lista de profissionais e seus respectivos locais de trabalho, que foi disponibilizada pelo gestor de cada serviço. No primeiro contato, foram explicados os objetivos da pesquisa e seus procedimentos, seguido do convite à participação. Nos casos de aceite, eram agendados dia, local e horário para a entrevista. Apenas um profissional não teve interesse em participar.

As entrevistas aconteceram nos serviços de saúde, locais de trabalho dos participantes, com duração média de 20 minutos. Após as mesmas, os questionários foram preenchidos pela pesquisadora, com o auxílio do participante. Todas as falas foram gravadas (com o consentimento do participante) e posteriormente transcritas. No que concerne às questões éticas, esta pesquisa foi submetida ao Comitê de Ética em Pesquisa com Seres Humanos (CEPSH) e foi aprovada em maio de 2013 (Parecer $n^{\circ} 275.875$, de 13/05/2013).

\section{Análise de Dados}

As entrevistas foram transcritas e a análise dos dados teve como suporte o programa Atlas/Ti 5.0", um software que trata e analisa dados qualitativos, auxiliando na organização e síntese de uma grande quantidade de material (Muhr, 2004). Na primeira parte da análise foi realizado o que Strauss e Corbin (2008) apresentam como codificação aberta, processo no qual "os conceitos são identificados e suas propriedades e dimensões são descobertas nos dados" (p. 103). Na sequência, procedeu-se à codificação axial, quando as categorias obtidas na codificação aberta foram relacionadas às subcategorias, chegando assim às respostas para os objetivos desta pesquisa (Strauss \& Corbin, 2008). A construção das categorias de análise seguiu o conceito de análise de conteúdo temática proposto por Bardin (1977). A construção das categorias aconteceu $a$ posteriori, à medida em que estas foram emergindo do texto durante a etapa de leitura flutuante e sendo interligadas às subcategorias, que por sua vez chegaram a um maior nível de codificação e desmembramento analógico, representado pelos elementos de análise.

\section{Resultados e discussão}

Este estudo teve a participação de $16^{1}$ profissionais de saúde de nível superior, dentre eles médicos, psicólogos, enfermeiros e assistentes sociais. Destes, 12 eram mulheres e quatro eram homens, 
com idades entre 25 e 50 anos. O tempo de exercício da profissão variou entre 8 meses e 27 anos e o tempo de trabalho em urgências e emergências foi de 8 meses a 29 anos de experiência. Quanto ao número de tentativas de suicídio atendidas, nove participantes declararam ter atendido entre $10 \mathrm{e}$ 50 tentativas, dois realizaram entre 51 e 100 atendimentos, dois estimaram entre 101 e 150 e três apontaram ter atendido mais de 151 tentativas de suicídio durante sua vida profissional. Ainda, 15 profissionais declararam nunca ter recebido capacitação por parte das instituições nas quais trabalham sobre o tema "suicídio".

Com o intuito de responder aos objetivos deste artigo, o acolhimento, atendimento e encaminhamentos realizados a pacientes atendidos em urgências e emergências por tentativas de suicídio foram organizados em três categorias de análise, observadas na Tabela 1.

\section{O Acolhimento por Tentativa de Suicídio}

O acolhimento realizado aos pacientes atendidos por tentativa de suicídio apresentou três aspectos: as características relacionadas às pessoas atendidas, as características relacionadas às tentativas de suicídio e a entrada do paciente na Unidade de saúde.
Entre as características relacionadas às pessoas atendidas, condições físicas e psicológicas foram descritas pelos participantes como comuns aos pacientes que chegaram aos serviços de saúde por tentativa de suicídio. Agitação, introspecção, lucidez e confusão mental foram exemplos apontados, que variaram de acordo com o método utilizado. Pacientes do sexo feminino foram atendidas com maior frequência, como é exemplificado na narrativa de P4, que afirmou que são "predominantemente mulheres, homens aqui acho que vi bem poucos, se eu vi algum são bem poucos, é predominantemente mulher ${ }^{2 \prime}$. Os dados também indicaram uma rede de apoio fragilizada do paciente, bem como a presença de relações conflitivas, seja com cônjuge, filhos ou outros familiares.

O predomínio do atendimento ao público feminino nos contextos de urgência e emergência por tentativa de suicídio vai ao encontro de estudos que apontaram nesta mesma direção (Pires, Raposo, Pires, Sougey, \& Bastos Filho, 2012; Pires, Silva, Passos, Sougey, \& Bastos Filho, 2014). Dada a letalidade dos métodos utilizados pelo público masculino - enforcamento, arma de fogo (Bando et al., 2012; Sá et al., 2010), muitas vezes não há tempo para atendimento nos serviços de saúde. Observou-se ainda consonância dos dados com

Tabela 1. Síntese das Categorias, Subcategorias e Elementos de Análise.

\begin{tabular}{|c|c|c|}
\hline Síntese das Categorias & Subcategorias & Elementos de análise \\
\hline \multirow[t]{3}{*}{$\begin{array}{l}\text { O acolhimento por tentativa de } \\
\text { suicídio }\end{array}$} & $\begin{array}{l}\text { Características relacionadas às pessoas } \\
\text { atendidas }\end{array}$ & $\begin{array}{l}\text { Condições físicas e psicológicas } \\
\text { Sexo feminino } \\
\text { Rede de apoio fragilizada } \\
\text { Relações conflitivas }\end{array}$ \\
\hline & $\begin{array}{l}\text { Características relacionadas às tentativas } \\
\text { de suicídio }\end{array}$ & $\begin{array}{l}\text { Falsa tentativa } \\
\text { Intoxicação } \\
\text { Acesso fácil a medicações }\end{array}$ \\
\hline & A entrada do paciente na unidade & $\begin{array}{l}\text { Diferentes formas de acesso } \\
\text { Triagem realizada pela equipe de enfermagem com classificação de risco }\end{array}$ \\
\hline \multirow[t]{2}{*}{$\begin{array}{l}\text { A atenção prestada às pessoas } \\
\text { atendidas por tentativa de suicídio }\end{array}$} & Procedimentos realizados & $\begin{array}{l}\text { Protocolo de atendimento de acordo com o método utilizado } \\
\text { Avaliação médica } \\
\text { Avaliação do CIT } \\
\text { Avaliação psicológica } \\
\text { Contato com o acompanhante/ } \\
\text { atendimento à família }\end{array}$ \\
\hline & $\begin{array}{l}\text { Dificuldades observadas no atendimento } \\
\text { ao paciente }\end{array}$ & $\begin{array}{l}\text { Inadequação da demanda ao serviço } \\
\text { Diferentes perspectivas de trabalho } \\
\text { Modelo compartimentalizado de atendimento } \\
\text { Limites na comunicação paciente/profissional } \\
\text { Ausência de médicos psiquiatras } \\
\text { Condições físicas do ambiente }\end{array}$ \\
\hline \multirow[t]{2}{*}{$\begin{array}{l}\text { Os encaminhamentos às pessoas } \\
\text { atendidas por tentativa de suicídio }\end{array}$} & Encaminhamentos feitos às instituições & $\begin{array}{l}\text { Unidades da rede municipal de saúde } \\
\text { Avaliação psiquiátrica/internação no instituto de psiquiatria } \\
\text { Rede não-governamental }\end{array}$ \\
\hline & Orientações à rede de apoio & Família \\
\hline
\end{tabular}


o estudo de Oliveira, Bezerra Filho e Gonçalves-Feitosa (2014), que indicou a presença de sentimento de rejeição de familiar ou pessoa próxima e ausência de amigos em $60 \%$ dos participantes. É consenso na literatura que isolamento social e conflitos familiares são fatores de risco importantes para tentativas de suicídio (Abreu, Lima, Kohlrausch, \& Soares, 2010; Loureiro, 2006).

Nas características relacionadas às tentativas de suicídio, as falsas tentativas, o acesso fácil às medicações e a intoxicação como método mais utilizado foram destaques. O P2, a seguir, apresentou o que entende como uma falsa tentativa:

Então eles dizem que ingeriram uma quantidade de um remédio que eles nem sabem qual, deixam as caixas espalhadas e você vai ver, na verdade nem tomaram nenhum às vezes, é mais pra chamar atenção, felizmente. Então a nossa amostra aqui, graças a Deus, as tentativas de suicídio não são reais. [...] Eles querem chamar atenção de alguma forma e aí usam a história da tentativa de suicídio pra chamar atenção (P2).

Dentre estas falsas tentativas, duas situações emergiram: na primeira, as tentativas praticadas pelos pacientes são percebidas como não reais, considerando o método utilizado e baixa intencionalidade de morte (os pacientes faziam uso de métodos cujos efeitos no organismo podiam ser revertidos através de cuidados médicos e, mediante entrevista clínica, apontavam que não tinham o desejo real de morte, mas sim de livrarem-se dos seus problemas). Para os participantes que corroboram essa ideia, cuja maioria não atuava com saúde mental ou não tinha formação na área (médicos, enfermeiros), o objetivo é terem a atenção de alguém, em especial uma pessoa de convivência próxima (esposo(a), namorado(a), filho(a). Segundo eles, estes pacientes vêm de contextos nos quais há intenso sofrimento psíquico decorrente de problemas familiares. $\mathrm{O}$ ato então externaliza uma dor psíquica ou um pedido de ajuda (Botega et al., 2009; Silva \& Boemer, 2004).

Na segunda situação, a tentativa é pouco valorizada pelos profissionais, que fazem uso de frases pejorativas como "não tentou da maneira certa" ou "a pessoa só quer chamar a atenção", entendendo que o ato é manipulativo e atrapalha a rotina do serviço. Relatos semelhantes são observados em outros estudos (Silva \& Boemer, 2004; Vidal \& Gontijo, 2013) e questionam o preparo profissional para lidar com estas situações (Abreu et al., 2010; Vidal \& Gontijo, 2013). O uso dos termos apresentados ainda ilustra o fato de que muitos dos pacientes que não conseguem elaborar o que sentem de forma organizada e enxergam o suicídio como única alternativa viável, costumam ser menosprezados ao invés de serem acolhidos nos serviços de saúde (Cassorla, 2006, como citado em Loureiro, 2006).

A despeito do significado que estes profissionais atribuem à tentativa de suicídio, faz parte do compromisso ético dos profissionais e dos órgãos públicos em proteger as pessoas que se encontram em situação de risco ou atentaram contra suas vidas, reconhecendo-as e acolhendo-as em suas diferenças (Daolio, 2012; Ministério da Saúde, 2010). Porém, diversos estudos apontam falta de preparo, de conhecimentos e preconceito dos profissionais de saúde para lidar com o paciente suicida (Freitas \& Martins-Borges, 2014; Kishi, Kurosawa, Morimura, Hatta, \& Thurber, 2011). Logo, à medida que estes reproduzem práticas e estigmas do senso comum, tem-se como consequência uma não procura ou a não-oferta de cuidado e de continuidade de atendimento, que traz consequências nos diferentes âmbitos da vida do paciente e mantém a condição de marginalizado e fracassado que a ele se imputa (Botega et al., 2009; Silva, Sougey, \& Silva, 2015).

Os dados indicam ainda que o fácil acesso às medicações e a intoxicação possuem relação estreita. A medicação da qual o paciente faz uso contínuo, seja para dor, problemas cardíacos ou transtornos mentais, é administrada em altas doses no momento da tentativa, o que leva a intoxicação a aparecer como o método mais comumente utilizado. Sá et al. (2010) mostraram que $63 \%$ dos pacientes atendidos num serviço de emergência por tentativa de suicídio chegaram por intoxicação e o principal método, utilizado por $70 \%$ dos pacientes, foi envenenamento intencional, corroboradas por outras pesquisas que também têm demonstrado um elevado percentual de tentativas de suicídio por intoxicação medicamentosa ou envenenamento (Agadir Santos et al., 2013; Pires et al., 2012).

Por fim, na entrada do paciente na Unidade, observaram-se diferentes formas de acesso (bombeiros, SAMU, trazido por familiares), ou seja, as formas como os pacientes chegaram ou foram trazidos aos serviços e a realização de triagem pela equipe de enfermagem com classificação de risco. P3 explica que os pacientes "... chegam já trazidos por equipe de referência, SAMU, bombeiro, ou já vindos encaminhados de outras unidades, outros postos de saúde, outros hospitais, de outros municípios". Com base nessa avaliação inicial, considerando as condições clínicas e sinais vitais, o paciente 
que tentou suicídio é classificado com risco alto e tem prioridade de atendimento, ou nos casos em que chega desacordado ou em situação de crise, vai direto para atendimento médico, que será direcionado pela tentativa realizada. Em todas as situações houve o acolhimento destes pacientes e na maioria dos casos, com atendimento prioritário em virtude do quadro clínico apresentado.

Observa-se, portanto, a necessidade da escuta qualificada e da construção de relações de confiança e de cuidado ainda no acolhimento dos serviços de urgência/emergência. Diferentes autores têm reiterado a importância da oferta de educação permanente aos profissionais que atuam nestes contextos para lidar com situações que desafiam as representações sobre a vida e a morte, para que a crença ou o julgamento não se imponha sobre a prática (Botega, 2015). Por fim, é por meio do acolhimento, enquanto estratégia balizadora da organização do SUS no Brasil, que será garantido o acesso e o referenciameto dos usuários aos serviços de saúde de maneira integral nos diferentes níveis de complexidade (Ministério da Saúde, 2010).

\section{A Atenção Prestada às Pessoas Acolhidas por Ten- tativa de Suicídio}

No atendimento prestado aos pacientes atendidos por tentativa de suicídio destacam-se os procedimentos realizados ao paciente após o acolhimento e as dificuldades que são encontradas neste processo. Nos serviços pesquisados, protocolos de atendimento orientavam o trabalho das equipes e variavam conforme o método utilizado na tentativa de suicídio. Havia um fluxo de atendimento que incluía avaliação médica, avaliação do CIT (Centro de Informações Toxicológicas) e avaliação psicológica, nos serviços em que o psicólogo fazia parte da equipe, ilustrado na fala de P4.

E [...], quando já é possível, o paciente já está estabilizado, entra a Psicologia. Psicologia, Serviço Social, porque muitas vezes esse paciente chega sozinho, não se sabe nem quanto de remédio que tomou, não se sabe nada, então isso é um fator que impede muitas vezes aqui na emergência, porque o paciente chega sem informação, então tu tem que seguir ali o que o paciente tá te apresentando. Então entra esse pessoal junto, tanto a equipe multidisciplinar, mas primeiro, entra a enfermagem e a Medicina sempre (P4).

Observa-se nesta fala a centralidade do modelo biomédico, cujo enfoque dos cuidados médicos se sobrepõe a um olhar ampliado de saúde. Silva (2005) defende que o hospital pode ser um espaço promotor da integralidade do cuidado e promoção de saúde, desde que saia da lógica médico-centrada para uma lógica usuário-centrada, na qual além dos cuidados técnicos e instrumentais, o usuário é um indivíduo de direitos e há a responsabilização pelo cuidado a partir do acolhimento e do vínculo. Práticas inovadoras não ganham espaço sem uma correlação de forças entre os atores envolvidos, contudo é preciso que se abandonem práticas ultrapassadas que já não dão mais conta das atuais necessidades humanas, estas biopsicossociais e culturais (Guedes \& Castro, 2009).

Entre as instituições participantes, apenas uma possuía psicólogos que atuavam na emergência e duas delas não apresentavam o profissional como parte da equipe técnica. No serviço que tinha psicólogo, o protocolo de atendimento instituía que, assim que o paciente estivesse clinicamente estável, fosse encaminhado ao Serviço de Psicologia. Neste atendimento, o profissional procurava coletar o maior número de informações sobre a história de vida do paciente e da tentativa, com o intuito de, ao fim da entrevista, ter condições de avaliar o risco de novas tentativas. Não se consegue prever se a pessoa tentará suicídio novamente; contudo, baseados no conhecimento dos fatores de risco e de proteção avaliados durante a entrevista clínica, é possível aferir o risco individual que o paciente apresenta naquele momento (Bertolote, Mello-Santos, \& Botega, 2010).

Também era rotina dos serviços realizar contato com uma pessoa de referência do paciente que estava sendo atendido, disponível para permanecer como acompanhante enquanto ele estivesse na unidade. $\mathrm{Na}$ chegada deste acompanhante, quando o contato com o paciente ainda não era possível devido as suas condições de saúde, era realizado atendimento à familia ou à rede de apoio. Este visava à coleta de maiores informações sobre a situação e a construção de um histórico de saúde, que incluía a presença de transtornos psiquiátricos ou tentativas de suicídio anteriores.

É importante esclarecer que os procedimentos realizados no atendimento ao paciente não se davam de forma linear, pois a atuação dos profissionais estava condicionada às condições apresentadas no momento. Assim, o atendimento ao acompanhante também era parte do processo de trabalho ao atender os pacientes acolhidos por tentativa de suicídio. Nestas situações, além destes encontrarem-se fragilizados, a família também está preocupada e assustada, com medo do que possa vir a acontecer, logo, mostra-se de grande 
importância a dedicação dos profissionais neste atendimento (Gutierrez, 2014), que refletirá no engajamento dos familiares nos encaminhamentos realizados.

Sobre as dificuldades encontradas na prestação do atendimento, os dados apontaram um questionamento dos participantes sobre a legitimidade das demandas de saúde mental para os serviços de urgência e emergência, referida como uma inadequação da demanda ao serviço e ilustrada por P10. Para ele, "o quadro psicológico, emocional, muitas vezes ele não é considerado uma demanda legítima de emergência, legítimo é infarto, AVC". Nesse sentido, outros relatos afirmaram que o ambiente das emergências não é adequado, portanto, o atendimento deveria ser realizado nas UBS, pois um serviço de alta complexidade destina-se ao atendimento de situações agudas e o paciente de saúde mental é um paciente que demanda muito das equipes, como trazido na narrativa de P5:

O problema é que aqui não é o ambiente adequado pra você abordar esse tipo de problema, a história é outra, [...] brigou com o namorado, o namorado falou que ia terminar, aí sabia que o namorado ia hoje passar por lá, daí inventou essa história. Assim, teria que encaminhar para UBS, pra ir lá e eles encaminharem pro psicólogo, então o problema é que tumultua, porque aqui [...] a nossa história é atender paciente com alguma patologia que a gente possa resolver, porque às vezes chega acidentado, baleado, [...] com corte, [...] dos bombeiros, clinicamente grave, então a abordagem é meio sumária, como a proposta [...] é tratar a coisa aguda, se não tem nada, a gente faz a pessoa ver que ela tá bem, ela tá bem, ela diz que tá bem, vai embora (P5).

Observou-se nos serviços de saúde a pouca aceitação e baixa operacionalização do cuidado em saúde mental nos contextos hospitalares, conforme proposto pela Reforma Psiquiátrica (Lei 10.216/2001). Máximo e Bosi (2006) avaliam que na organização do processo de trabalho dos serviços de emergência, os critérios tempo e produtividade levam quase a uma "solução de descarte", na qual o objetivo passa a ser dar respostas rápidas e quase sem contato. Logo, as práticas em saúde existentes nas emergências não respondem às necessidades dos pacientes de saúde mental, que requerem maior disponibilidade por parte dos profissionais. Contudo, Gutierrez (2014) afirma que é possível promover qualidade de vida a pacientes que tentaram suicídio e dar conta de parte dos problemas presentes na tentativa de suicídio ainda no ambiente hospitalar, por meio da integralidade do cuidado. Segundo a autora, quando o profissional de saúde se coloca no lugar do paciente, ele consegue ofertar atendimento de qualidade, que deixa a mecanização de lado e oferta o seu melhor para o outro (Gutierrez, 2014).

Foram verificadas também diferentes perspectivas de trabalho, que em alguns momentos reforçaram um modelo compartimentalizado de atendimento, no qual cada classe profissional faz a sua parte e não se comunica com as demais para um trabalho em parceria. Vidal e Gontijo (2013) pontuam que os profissionais de saúde que atendem às tentativas de suicídio em emergências hospitalares têm um papel importante no aumento ou decréscimo das taxas de mortalidade. Logo, a assistência que é prestada deve considerar que o paciente possui necessidades de saúde e que naquele momento tem necessidades hospitalares. Isso deve orientar os profissionais a um cuidado integral que extrapole a cura de uma doença e considere também que os cuidados em saúde incluirão acabar com as causas, tratar os danos e diminuir os riscos (Gutierrez, 2014).

Outra dificuldade encontrada refere-se às limitações de comunicação entre paciente e profissional de saúde, seja porque o paciente está sem condições físicas de falar (sedado, sonolento, agitação psicomotora) ou porque escolhe deliberadamente a não comunicação com os profissionais. Contudo é neste momento, quando a pessoa nem sempre está disposta a colaborar, está fraca ou agindo com hostilidade que o profissional precisa buscar estabelecer um bom vínculo (Botega et al., 2009). Este vínculo é o que vai garantir a confiança do paciente no profissional e sua participação efetiva no atendimento, bem como uma escuta e uma adequada avaliação de risco.

A ausência de médicos psiquiatras nos serviços e as condições estruturais também dificultam os atendimentos, já que a consulta psiquiátrica se dá via pedido de parecer, que tem até 48 horas para ser respondido e os atendimentos acontecem nas instalações dos serviços, não sendo sempre possível garantir sigilo e privacidade aos pacientes. Destacaram-se, por fim, a compartimentalização dos saberes dentro do ambiente hospitalar, o saber psi e o saber não psi.

A fala de P9 problematizou esta situação, ao descrever que "... de escuta qualificada, acaba ficando com a Psicologia, a Medicina, ele entende assim, nós fazemos a parte clínica, a Psicologia faz essa parte". Maciel (1994) lembra que, no pensamento científico ocidental, há uma fragmentação de domínios que reflete nas 
terapêuticas em saúde. Logo, são claramente identificados os profissionais que cuidam do corpo que está doente, o corpo físico e os que cuidam dos sintomas da alma, os profissionais psi. Esta dualidade tem um impacto importante sobre os atendimentos. Estudos têm demonstrado diferentes significados atribuídos às tentativas de suicídio por profissionais com formação ou especialistas em saúde mental e os que não o são (Freitas \& Martins-Borges, 2014; Silva \& Boemer, 2004), bem como indicam atitudes mais positivas junto aos pacientes por parte dos profissionais com formação ou especialização em saúde mental (Kishi et al., 2011; Srivastava \& Tiwari, 2012).

Há que se destacar que a permanência do paciente no serviço de saúde é um momento importante para dar apoio e ajudá-lo na busca de um novo significado à sua vida, estimulando-o a desenvolver maior autonomia (Gutierrez, 2014) e uma equipe que apresenta atitudes acolhedoras e respeitosas tem maiores chances de êxito nessa tarefa. Evidenciou-se então neste estudo, a partir dos relatos e do número de participantes que relatou ausência de capacitação institucional, a necessidade de instrumentalização para lidar com as tentativas de suicídio do cotidiano das urgências e emergências hospitalares, demanda esta também assinalada por outros estudos realizados nestes mesmos contextos (Gibb, Beaustrais, \& Surgenor, 2010; Gutierrez, 2014; Vidal \& Gontijo, 2013).

\section{Os Encaminhamentos Pós-Alta Realizados às Pes- soas Atendidas por Tentativa de Suicídio}

A realização de encaminhamentos pós-alta foi uma prática encontrada em todos os serviços de saúde pesquisados. Ela se dá via encaminhamentos a outras instituições ou por meio de orientações à rede de apoio. Nos encaminhamentos feitos às instituições, destacaram-se os encaminhamentos dirigidos às Unidades da Rede Municipal de Saúde, à avaliação psiquiátrica ou à internação no Instituto de Psiquiatria e os encaminhamentos à rede não governamental. Já as orientações à rede de apoio foram direcionadas às famílias e à rede de apoio próxima.

A Rede Municipal de Saúde configura-se como fonte primária de encaminhamentos dos pacientes após alta hospitalar, com duas opções importantes. A primeira são as Unidades Básicas de Saúde (UBS), onde o paciente poderá dar continuidade ao seu tratamento próximo à sua residência. Uma segunda opção são os CAPS (Centro de Atenção Psicossocial), equipamentos da atenção secundária. Contudo, se o paciente mantiver ideação suicida ou oferecer risco a si mesmo ou a outras pessoas, a conduta adotada é encaminhá-lo para avaliação psiquiátrica ou internação no Instituto de Psiquiatria da região.

É salutar a inclusão da avaliação do risco de novas tentativas de suicídio na rotina dos serviços de urgência e emergência antes que o paciente receba alta, a fim de que o encaminhamento proposto seja condizente com suas necessidades (Bertolote et al., 2010). Isso requer que a equipe de saúde tenha competência técnica e utilize os instrumentos adequados para avaliar todas as vulnerabilidades do paciente enquanto ele estiver no ambiente hospitalar. Condições físicas, psicológicas, sociais e recursos disponíveis de saúde no território são variáveis que precisam ser investigadas (Gutierrez, 2014), contudo, os profissionais assinalaram dificuldades para concretizar os encaminhamentos, pontuando que as opções disponíveis na rede são escassas frente à demanda atendida.

As orientações direcionadas aos familiares ou à pessoa de referência buscaram implicá-los nos encaminhamentos realizados pelos profissionais quando o paciente deixa o serviço de saúde. As orientações vão desde administrar as medicações que o paciente faz uso, para que não haja nova intoxicação, deixar fora de alcance objetos cortantes ou armas de fogo, até acompanhá-lo aos locais para dar seguimento ao seu tratamento, como as consultas no CAPS. Sobre esse processo, P4 afirma que "... [quando] esse paciente acaba indo pra casa, com as orientações de no outro dia buscar por conta própria, [...] se o cara tentou se matar depois ele não vai por conta própria".

Reforçando a importância da família nesse processo, o estudo de Sá et al. (2010) apontou que 45,2\% dos pacientes atendidos em emergência hospitalar após tentativa de suicídio receberam alta hospitalar, 36,6\% ficaram internados e 11,6\% foram encaminhados para tratamento ambulatorial. Avalia-se então que a adequada instrução da família auxilia na prevenção do suicídio. Nunca deixar o paciente sozinho, informar demais familiares sobre a tentativa praticada, para que possam ficar vigilantes, mas ao mesmo tempo oferecer suporte emocional, e procurar atendimento especializado nos serviços de saúde mental são orientações importantes (Barrero, 2008).

Uma observação importante sobre os encaminhamentos referiu-se aos serviços sem psicólogo na equipe técnica. Nestes casos, os profissionais de saúde encaminhavam os pacientes e familiares para o Serviço 
Social, responsável pelo encaminhamento institucional e pelas demais orientações. P15 descreveu que "agora que a gente às vezes tem até o Serviço Social, o que a gente tenta fazer é manter o paciente aqui até a chegada do Serviço Social durante o dia, para que ele possa tomar o devido encaminhamento". Ainda, complementou P12:

... Trata a questão clínica e encaminha para a assistente social caso ela vá estar aqui no outro dia de manhã e final de semana é difícil, porque aí não tem Caps, não tem serviço social, então esse paciente acaba indo pra casa, com as orientações de no outro dia buscar por conta própria (P12).

Contudo, este encaminhamento nem sempre é possível. A decisão sobre o paciente permanecer no serviço até o dia seguinte é uma decisão médica. Já, nos finais de semana, com exceção do Instituto de Psiquiatria da região, não há nenhum outro serviço de saúde mental que funcione. Essa é uma situação delicada, pois é alto o número de pacientes que tentaram suicídio e têm diagnóstico de transtorno mental e precisam de acompanhamento especializado (Vidal \& Gontijo, 2013). Reitera-se assim a necessidade de um trabalho de equipe interdisciplinar, no qual as decisões sejam coletivas e os saberes das diferentes áreas de conhecimento sejam complementares. A interdisciplinaridade, somada à articulação com a rede de serviços disponíveis no território, trazem bons indicativos para a oferta de um cuidado integral às pessoas que tentaram suicídio (Gutierrez, 2014; Sá et al., 2010).

\section{Conclusões}

$\mathrm{O}$ adequado atendimento aos pacientes que chegam aos serviços de saúde por tentativa de suicídio é determinante para a recuperação e prevenção de que novas tentativas sejam cometidas. Considerando tal importância, o presente artigo investigou como se dá o atendimento a pacientes que tentaram suicídio desde sua entrada até a saída dos serviços de urgência ou emergência hospitalar, em um município do sul do Brasil.

Os dados mostraram que, no acolhimento, a demanda atendida nos serviços de urgência e emergência pesquisados não difere do perfil atendido em outros serviços de saúde semelhantes, conforme descreve a literatura. Destacaram-se o predomínio do público feminino, a intoxicação como método mais utilizado e a presença de relações ou contextos conturbados e/ou instáveis que funcionam como desencadeadores para as tentativas.

Ainda, as tentativas intituladas pelos participantes como tentativas de chamar a atenção, nomeadas também de falsas tentativas de suicídio, foram descritas como as demandas de maior frequência nos atendimentos e apontadas como uma das dificuldades presentes no atendimento ao paciente. Segundo eles, demandas de atendimento desencadeadas pelo sofrimento psíquico, como as tentativas de suicídio, atrapaIham o trabalho em saúde que é realizado em urgências ou emergências hospitalares.

No atendimento, ainda que organizado de forma singular em cada local pesquisado, os dados evidenciaram que os serviços de saúde dispõem de protocolos mínimos para atendimento ao paciente que chega às unidades por tentativa de suicídio, sendo esta demanda absorvida pela rotina do serviço. São retomados também no atendimento, aspectos já apontados na categoria anterior, o acolhimento, como a inadequação da demanda aos serviços e diferentes olhares sobre o fenômeno do suicídio, cuja explicação pode ter relação com a formação de cada profissional. Contudo, a principal diferença observada é a presença do Serviço de Psicologia em uma das unidades, o que facilitava o atendimento e os encaminhamentos para o paciente após a alta hospitalar.

E por fim, sobre os encaminhamentos, foi possível perceber que estes ocorrem baseados na avaliação de risco feita pelos profissionais. Contudo, ainda que haja um engajamento nesta tarefa de garantir um acoIhimento ou a continuidade do tratamento do paciente na rede, foram assinaladas fragilidades no sistema de saúde, como poucas opções disponíveis para encaminhamento deste público específico e consequente dificuldade de absorção de toda a demanda.

Ainda que os resultados apresentados tenham validade para as condições e contexto de pesquisa expostos, este estudo contribuiu com o debate sobre a necessidade de capacitação para o manejo das situações de tentativas de suicídio, aos profissionais de saúde que atuam nos contextos de urgências e emergências hospitalares. Torna-se salutar que os conteúdos teórico-práticos sobre saúde mental, com destaque para a discussão do comportamento suicida, sejam incluídos nos currículos dos cursos da área da Saúde.

Reitera-se também a importância do trabalho interdisciplinar para a oferta de cuidado integral, importante para a prevenção de novas tentativas e promoção de qualidade de vida dos pacientes. Isso implica tornar 
o atendimento às pessoas que tentam suicídio mais qualificado e menos atravessado por preconceitos e/ ou julgamentos morais, já que os dados mostraram que as tentativas, em muitos casos, são entendidas como afronta à prática profissional. Destaca-se que a empatia e o vínculo são fundamentais ao atendimento em saúde e perceber o sofrimento psíquico vivenciado pelo paciente, subjacente à tentativa de suicídio possibilita oferecer cuidados mais acolhedores e menos punitivos.

Entre as limitações encontradas, observou-se que nem sempre foi possível a realização das entrevistas em ambientes sem interrupções, já que as mesmas foram realizadas nos locais de trabalho dos participantes. A interrupção de algumas entrevistas pode ter trazido perdas no discurso ou ainda a quebra do raciocínio que vinha sendo desenvolvido. É também limitação deste estudo a não realização de uma análise mais aprofundada que diferencie cuidados ofertados por profissionais com formação ou especialização em saúde mental e os que não o são. Isso indica a necessidade da realização de novas pesquisas sobre o cuidado e formação profissional no atendimento a tentativas de suicídio, considerando todavia que a temática está longe de ser esgotada.

\section{Referências}

Abreu, K. P, Lima, M. A. D., Kohlrausch, E., \& Soares, J. F. (2010). Comportamento suicida: fatores de risco e intervenções preventivas. Revista eletrônica de Enfermermagem, 12(1), 195-200. doi: 10.5216/ree.v12i1.9537

Agadir Santos, A. S., Legay, L. F., \& Lovisi, G. M. (2013). Substâncias tóxicas e tentativas e suicídios: considerações sobre acesso e medidas restritivas. Cadernos Saúde Coletiva, 21(1), 53-61. doi: 10.1590/S1414-462X2013000100009

Bando, D. H., Brunoni, A. R., Fernandes, T. G., Benseñor, I. M., \& Lotufo, P. A. (2012). Suicide rates and trends in São Paulo, Brazil, according to gender, age and demographic aspects: a joinpoint regression analysis. Revista Brasileira de Psiquiatria, 34(3), 286-293. doi: 10.1016/j.rbp.2012.02.001

Bardin, L. (1977). Análise de conteúdo. Lisboa: Edições 70.

Barrero, S. A. P. (2008). Preventing suicide: a resource for the family. Annals of General Psychiatry, 7(1). doi:10.1186/1744-859X-7-1

Bertolote, J. M., Mello-Santos, C., \& Botega, N. J. (2010). Detecção do risco de suicídio nos serviços de emergência psiquiátrica. Revista Brasileira de Psiquiatrira, 32, 87-95. doi: 10.1590/ S1516-44462010000600005

Botega, N. J (2015). Crise suicida: avaliação e manejo. Porto Alegre, Artmed, 2015.

Botega, N. J., Marín-León, L., Oliveira, H. B., Barros, M. B. A., Silva, V. F., \& Dalgalarrondo, P. (2009). Prevalências de ideação, plano e tentativa de suicídio: um inquérito de base populacional em Campinas, São Paulo, Brasil. Cadernos de Saúde Pública, 25(12), 2632-2638. doi: 10.1590/S0102-311X2009001200010
Daolio, E. R. (2012). Suicídio: tema de reflexão bioética. Revista Bioética, 436-441. Recuperado de http://revistabioetica.cfm.org.br/ index.php/revista_bioetica/article/view/763

Freitas, A. P. A., \& Martins-Borges, L. (2014). Tentativas de suicídio e profissionais de saúde: significados possíveis. Estudos e Pesquisas em Psicologia, 14(2), 560-577. Recuperado de http://pepsic.bvsalud.org/scielo.php?script=sci_arttext\&pi$\mathrm{d}=$ S1808-42812014000200010\&lng=pt\&tIng=pt

Gibb, S. J.; Beautrais, A. L., \& Surgenor, L. J. (2010). Healthcare staff attitudes towards self-harm patients. Australian and New Zealand Journal of Psychiatry. 44, 713-720. doi: 10.3109/00048671003671015

Guedes, H. H. S., \& Castro, M. M. C. (2009). Atenção hospitalar: um espaço produtor do cuidado integral em saúde. Serviço Social em Revista, 12(1), 4-26. doi: 10.5433/1679-4842.2009v12n1p4

Gutierrez, B. (2014). Assistência hospitalar na tentativa de suicídio. Psicologia USP, 25(3), 262-269. doi: 10.1590/0103-6564D20140002

Kishi, Y.; Kurosawa, H.; Morimura, H.; Hatta, K.; \& Thurber, S. (2011). Attitudes of Japanese nursing personnel toward patients who have attempted suicide. General Hospital Psychiatry, 33, 393-397. doi: 10.1016/j.genhosppsych.2011.02.005

Lei no 10.216, de 6 de abril de 2001 (2001, 9 de abril). Dispõe sobre a proteção e os direitos das pessoas portadoras de transtornos mentais e redireciona o modelo assistencial em saúde mental. Diário Oficial da União, seção 1. Recuperado de http://www.planalto.gov. br/ccivil_03/leis/leis_2001/110216.htm

Loureiro, R. M. (2006). Um possível olhar do comportamento suicida pelos profissionais de saúde. Scientia Medica, 16(2). Recuperado de http://revistaseletronicas.pucrs.br/ojs/index.php/scientiamedica/ article/view/1622

Maciel, C. L. C. (1994). Emoção, doença e cultura: o caso da hipertensão arterial. In B. V. Romano (Org.), A prática da psicologia nos hospitais. São Paulo, SP: Livraria Pioneira.

Magalhães, A. P. N., Alves, V. M., Comassetto, I., Lima, P. C., Faro, A. C. M., \& Nardide, A. E. (2014). Atendimento a tentativas de suicídio por serviço de atenção pré-hospitalar. Jornal Brasileiro de Psiquiatria, 63(1),16-22. http://dx.doi.org/10.1590/0047-2085000000003.

Máximo, H. R. M., \& Bosi, M. L. M. (2006). Saúde mental em um hospital público: o olhar de profissionais médicos do município do Rio de Janeiro. Physis: Revista de Saúde Coletiva, 16(2), 293-316. doi: 10.1590/S0103-73312006000200009

Ministério da Saúde. Secretaria de Atenção à Saúde. Política Nacional de Humanização da Atenção e Gestão do SUS (2009). Acolhimento e classificação de risco nos serviços de urgência. Série B. Textos Básicos de Saúde. Brasília: Ministério da Saúde.

Ministério da Saúde. Secretaria de Atenção à Saúde. Núcleo Técnico da Política Nacional de Humanização (2010). Acolhimento nas práticas de produção de saúde (2 $2^{\underline{a}}$ ed., $5^{\text {a }}$. Reimpressão). Brasília: Editora do Ministério da Saúde.

Ministério da Saúde (2012). Política Nacional de Atenção Básica. Brasília: Editora do Ministério da Saúde. Recuperado de http://189.28.128.100/dab/docs/publicacoes/geral/pnab.pdf

Ministério da Saúde (2013). Saúde Brasil: Uma análise da situação de saúde e das doenças transmissíveis relacionadas à pobreza. Brasília: Editora do Ministério da Saúde. Recuperado de http://bvsms. saude.gov.br/bvs/publicacoes/saude_brasil_2013_analise_situacao_saude.pdf 
Do acolhimento ao encaminhamento: $\mathrm{O}$ atendimento às tentativas de suicídio nos contextos hospitalares

Muhr, T. (2004). Atlas/ti the knowledge workbench. V 5.0 Quick tour for beginners. Berlin: Scientific Software Development.

Nock, M. K., Borges, G., Bromet, E. J., Cha, C. B., Kessler, R. C., \& Lee, S. (2008). Suicide and suicidal behavior. Epidemiologic Reviews, 30(1), 133-154. doi: 10.1093/epirev/mxn002

Oliveira, M. I., Bezerra Filho, J. G., \& Gonçalves-Feitosa, R. F. (2014). Tentativas de suicídio atendidas em unidades públicas de saúde de Fortaleza-Ceará, Brasil. Revista de Salud Pública, 16(5), 687-699. doi: 10.15446/rsap.v16n5.40138

Organização Mundial de Saúde (2000). Prevenção do suicídio: um manual para médicos clínicos gerais. Genebra: Autor.

Pires, M. C. C., Raposo, M. C. F., Pires, M., Sougey, E. B., \& Bastos Filho, O. C. (2012). Stressors in attempted suicide by poisoning: a sex comparison. Trends in Psychiatry and Psychotherapy, 34(1), 25-30. doi: 10.1590/S2237-60892012000100006

Pires, M. C. C., Silva, T. P.S., Passos, M. P., Sougey, E. B. O., \& Bastos Filho, O. C. (2014). Fatores de risco para tentativa de suicídio por envenenamento: revisão. Trends in Psychiatry and Psychotherapy, 36(2), 63-74. doi: 10.1590/2237-6089-2013-0044

Resolução no 196 do Conselho Nacional de Saúde (1996, 10 de outubro). Dispõe sobre diretrizes e normas regulamentadoras de pesquisas envolvendo seres humanos. Brasília, DF: Ministério da Saúde. Recuperado de http://bvsms.saude.gov.br/bvs/saudelegis/ cns/1996/res0196_10_10_1996.html

Sá, N. N. B., Oliveira, M. G. C., Mascarenhas, M. D. M., Yokota, R. T. C., Silva, M. M. A., \& Malta, D. C. (2010). Atendimentos de emergência por tentativas de suicídio, Brasil, 2007. Revista Médica de Minas Gerais, 20(2), 145-152. Recuperado de http://www.rmmg. org/artigo/detalhes/307

Silva, T. P. S., Sougey, E. B., \& Silva, J. (2015). Estigma social no comportamento suicida: reflexões bioéticas. Revista Bioética, 23(2),
419-426. Recuperado de http://revistabioetica.cfm.org.br/index. php/revista_bioetica/article/view/1051

Silva, V. P., \& Boemer, M. R. (2004). O suicídio em seu mostrar-se a profissionais de saúde. Revista Eletrônica de Enfermagem, 6(2), 143-152. Recuperado de http://www.revistas.ufg.br/index.php/fen

Simonetti, A. (2004). Manual de psicologia hospitalar: o mapa da doença. São Paulo, SP: Casa do Psicólogo.

Srivastava, M., \& Tiwari R. (2012). A comparative study of attitude of mental health versus nonmental health professionals toward suicide. Indian Journal of Psychological Medicine, 34, 66-69. doi:10.4103/0253-7176.96163

Stefanello, B., \& Furlanetto, L. M. (2012). Ideação suicida em pacientes internados em enfermarias de clínica médica: prevalência e sintomas depressivos associados. Jornal Brasileiro de Psiquiatria, 61(1), 2-7. Recuperado de http://www.scielo.br/pdf/jbpsiq/ v61n1/02.pdf

Strauss, A., \& Corbin, J. (2008). Pesquisa qualitativa. Técnicas e procedimentos para o desenvolvimento de teoria fundamentada. Porto Alegre: Artmed.

Vidal, C. E. L., \& Gontijo, E. D. (2013). Tentativas de suicídio e o acolhimento nos serviços de urgência: a percepção de quem tenta. Cadernos Saúde Coletiva, 21(2), 108-114. doi: 10.1590/ S1414-462X2013000200002

Vidal, C. E. L., Gontijo, E. C. D. M., \& Lima, L. A. (2013). Tentativas de suicídio: fatores prognósticos e estimativa do excesso de mortalidade. Cadernos de Saúde Pública, 29(1), 175-187. doi: 10.1590/ S0102-311X2013000100020

Waiselfisz, J. J. (2011). Mapa da Violência 2011: os jovens do Brasil. Brasília, DF: Ministério de Justiça, Instituto Sangari. Recuperado de http://mapadaviolencia.org.br/pdf2011/acidentes_transito.pdf.

\footnotetext{
1. Os participantes são identificados como P1, P2, P3... P16.
}

2. As narrativas dos participantes foram utilizadas em sua forma original, sem correções gramaticais ou de concordância.

Ana Paula Araújo de Freitas, Mestre em Psicologia pela Universidade Federal de Santa Catarina (UFSC).

Endereço para correspondência: Universidade Federal de Santa Catarina, Centro de Filosofia e Ciências Humanas, Departamento de

Psicologia, Sala 8B

Campus Universitário - Trindade, Florianópolis - SC - CEP: 88.040-500. E-mail: anapfreitas2812@gmail.com

Lucienne Martins Borges, Doutorado em Psicologia pela Université du Québec à Trois-Rivières, Canadá, Pós-doutorado em Psicologia pela Université du Québec à Montréal, Canadá, é Professora do Departamento de Psicologia da Universidade Federal de Santa Catarina (UFSC). E-mail: lucienne.borges@ufsc.br 